\title{
BMJ Open Global epidemiology of hip fractures: a study protocol using a common analytical platform among multiple countries
}

Chor-Wing Sing (D) , ${ }^{1}$ Tzu-Chieh Lin, ${ }^{2}$ Sharon Bartholomew, ${ }^{3} \mathrm{~J}$ Simon Bell, ${ }^{4}$ Corina Bennett, ${ }^{2}$ Kebede Beyene, ${ }^{5}$ Pauline Bosco-Lévy, ${ }^{6}$ Amy Hai Yan Chan, ${ }^{5}$ Manju Chandran, ${ }^{7}$ Ching-Lung Cheung, ${ }^{1}$ Caroline $Y$ Doyon, ${ }^{3}$ Cécile Droz-Perroteau, ${ }^{6}$ Ganga Ganesan, ${ }^{8}$ Sirpa Hartikainen, ${ }^{9}$ Jenni Ilomaki, ${ }^{4}$ Han Eol Jeong, ${ }^{10}$ Douglas P Kiel, ${ }^{11}$ Kiyoshi Kubota, ${ }^{12}$ Edward Chia-Cheng Lai, ${ }^{13}$ Jeff Lange, ${ }^{2}$ E Michael Lewiecki, ${ }^{14}$ Jiannong Liu, ${ }^{15}$ Kenneth K C Man (D) , ${ }^{16,17}$ Mirhelen Mendes de Abreu (D) , ${ }^{18}$ Nicolas Moore, ${ }^{6}$ James O'Kelly, ${ }^{2}$ Nobuhiro Ooba, ${ }^{19}$ Alma B Pedersen, ${ }^{20}$ Daniel Prieto-Alhambra (D) , ${ }^{21}$ Ju-Young Shin (D) , ${ }^{10}$ Henrik T Sørensen, ${ }^{20}$ Kelvin Bryan Tan, ${ }^{8,22}$ Anna-Maija Tolppanen, ${ }^{9}$ Katia M C Verhamme (D) ,23 Grace Hsin-Min Wang, ${ }^{13}$ Sawaeng Watcharathanakij, ${ }^{24}$ Hongxin Zhao (D) , ${ }^{25}$ Ian C K Wong ${ }^{1,16,17}$

To cite: Sing C-W, Lin T-C, Bartholomew S, et al. Global epidemiology of hip fractures: a study protocol using a common analytical platform among multiple countries. BMJ Open 2021;11:e047258. doi:10.1136/ bmjopen-2020-047258

- Prepublication history and supplemental material for this paper is available online. To view these files, please visit the journal online. (http://dx.doi. org/10.1136/bmjopen-2020047258).

Received 24 November 2020 Accepted 05 July 2021

Check for updates

(C) Author(s) (or their employer(s)) 2021. Re-use permitted under CC BY-NC. No commercial re-use. See rights and permissions. Published by BMJ.

For numbered affiliations see end of article.

Correspondence to

Dr lan C K Wong;

wongick@hku.hk

\section{ABSTRACT}

Introduction Hip fractures are associated with a high burden of morbidity and mortality. Globally, there is wide variation in the incidence of hip fracture in people aged 50 years and older. Longitudinal and cross-geographical comparisons of health data can provide insights on aetiology, risk factors, and healthcare practices. However, systematic reviews of studies that use different methods and study periods do not permit direct comparison across geographical regions. Thus, the objective of this study is to investigate global secular trends in hip fracture incidence, mortality and use of postfracture pharmacological treatment across Asia, Oceania, North and South America, and Western and Northern Europe using a unified methodology applied to health records.

Methods and analysis This retrospective cohort study will use a common protocol and an analytical common data model approach to examine incidence of hip fracture across population-based databases in different geographical regions and healthcare settings. The study period will be from 2005 to 2018 subject to data availability in study sites. Patients aged 50 years and older and hospitalised due to hip fracture during the study period will be included. The primary outcome will be expressed as the annual incidence of hip fracture. Secondary outcomes will be the pharmacological treatment rate and mortality within 12 months following initial hip fracture by year. For the primary outcome, crude and standardised incidence of hip fracture will be reported. Linear regression will be used to test for time trends in the annual incidence. For secondary outcomes, the crude mortality and standardised mortality incidence will be reported.

Ethics and dissemination Each participating site will follow the relevant local ethics and regulatory frameworks for study approval. The results of the study will be submitted for peer-reviewed scientific publications and presented at scientific conferences.

\section{Strengths and limitations of this study}

- This study will involve countries/regions across Asia, Oceania, North and South America, Western and Northern Europe.

- The study will use a common protocol and an analytical common data model to ensure consistency in data analysis and validity in cross-geographical comparisons.

- This study will build a global real-world data platform to efficiently collaborate across multiple institutions.

- Several databases will capture only treatments in the public reimbursement system. Hence, the treatment rates might be underestimated by not including patients in the private payment system.

- Though most of the data sources will be representative of the country-specific population, a few data sources will be representative of local hospitals and regional population.

\section{INTRODUCTION}

Hip fracture is a leading cause of high morbidity $(30 \%-50 \%$ of patients lose functional independence $)^{1}{ }^{2}$ and mortality (approximately $22 \%$ mortality rate at 1 year). ${ }^{3}$ Globally, there is wide variation in the incidence of hip fracture in people aged 50 years and older, ${ }^{4}$ ranging from an age-standardised rate of over 500 cases per 100000 adults (eg, Denmark) to less than 100 cases per 100000 adults (eg, South Africa). Secular trends in the incidence of hip fracture have been suggested to follow the level of urbanisation. ${ }^{1}$

Following a hip fracture, individuals are at greater risk of another osteoporotic fracture 
relative to those without a fracture. For example, in a study that included over 96000 U.S. postmenopausal women who sustained a hip fracture, $8 \%$ had another clinical fracture within 1 year, $15 \%$ within 2 years, and $25 \%$ within 5 years. ${ }^{5}$ To reduce the risk of a subsequent fracture, clinical guidelines from American and European societies for bone and osteoporosis recommend pharmacological treatment to reduce fracture risk after a hip fracture ${ }^{67}$ Irrespective of guidelines, treatment rates in postfracture populations have been reported to be low in several geographical regions $(16 \%-21 \%$ of patients received pharmacological treatment $)^{89}$ and appear to be decreasing in both the U.S. ${ }^{10}$ and Europe. ${ }^{11}$ Given that pharmacological treatments have demonstrated a $30 \%-50 \%$ reduction in subsequent fracture,${ }^{12}$ many fractures occurring now are preventable. ${ }^{13}$

Longitudinal and cross-geographical comparisons of health data can provide insights on aetiology, risk factors, and healthcare practices. However, global reports are typically systematic literature reviews based on studies representing a heterogeneity of methods and study periods, making it a challenge to examine and compare data between geographical regions. For hip fracture specifically, the current available reports on hip fracture incidence are based on 20-year-old data in some geographical regions. ${ }^{14}$ Thus, we will investigate the global secular trends in hip fracture for incidence, mortality, and use of postfracture pharmacological treatment across Asia, Oceania, North and South America, Western and Northern Europe using a unified methodology applied to health records.

This study will use a common protocol and an analytical common data model (ACDM) approach to examine incidence of hip fracture using population-based databases from different geographical regions and healthcare settings. The concept of ACDM is to standardise a limited set of extracted variables into a common data structure, allowing the use of common analytics and methods across multiple datasets. ${ }^{15}$ Thus, the quality of data analyses in each study site can be controlled by using standardised methodologies including definition, calculation, and standardisation. This approach will provide high quality and comparable data on hip fracture and, therefore, is superior to data from systematic reviews of individual studies that have applied diverse methodologies. ${ }^{14}$ The standardisation of estimates can facilitate cross-geographical comparisons. In addition, this study will build a global real-world data platform to efficiently collaborate across multiple institutions.

\section{Hypothesis and objectives}

This is an estimation study and no hypothesis will be tested. The study aim is to characterise hip fracture incidence estimates by year and assess the trend among men and women aged 50 years and older within multiple countries. We aim to investigate the between-country and between-region differences in hip fracture incidence, mortality and pharmacological treatment rate. This may in turn lead to research into environmental, sociodemographic and biological explanatory factors for geographical variations in incidence and mortality of hip fracture.

Primary objective

- To estimate the annual incidence of hip fracture and evaluate the trend during 2005-2018 (objective 1).

Secondary objective

- To estimate the proportion of patients using a pharmacological treatment for osteoporosis within 12 months following their initial hip fracture by calendar year (objective 2).

- To estimate the mortality rate within 12 months following patients' initial hip fracture by calendar year (objective 3).

\section{Methods and analysis}

The study is in the common data model development phase. We plan to start the data analysis in the second quarter of 2021. The study will end in the first quarter of 2022.

\section{Study design}

This is a retrospective cohort study based on healthcare databases from multiple sites representing numerous geographical regions. To enable consistent analysis and reporting across different databases in different regions and healthcare settings, a common protocol, statistical analysis plan, and an ACDM will be used to obtain aggregated data from each database. The study will consist of annual cohorts of patients who experience hip fracture from each database. Each site will convert their raw data into an ACDM format and apply the common statistical code provided by the study coordinator (University of Hong Kong, HKU) to perform the analysis. The study coordinator will not receive any patient-level data from the sites. Instead, each site will conduct the analysis locally using a centrally developed analytic plan and share aggregated results with the study coordinator for the analysis of the pooled data.

\section{Data source}

This study will obtain aggregated data from the participating sites. All included sites will use patient-level electronic health data derived from the respective national or regional administrative databases, clinical databases, or registry databases. The study period will be from 1 January 2005 to 31 December 2018, subject to data availability in each study site. A full list of participating sites and databases is provided in table 1.

The study sites will contribute aggregated data on diagnosis, medications, mortality and other data associated with hip fracture in a defined population. Depending on the data capability to address study questions (ie, fit-forpurpose), the study sites will contribute aggregated data for some or all of the objectives. Study sites can contribute incidence estimates for objective 1 for data sources of population-based data (ie, a defined denominator). If complete prescription data are available, study sites can 


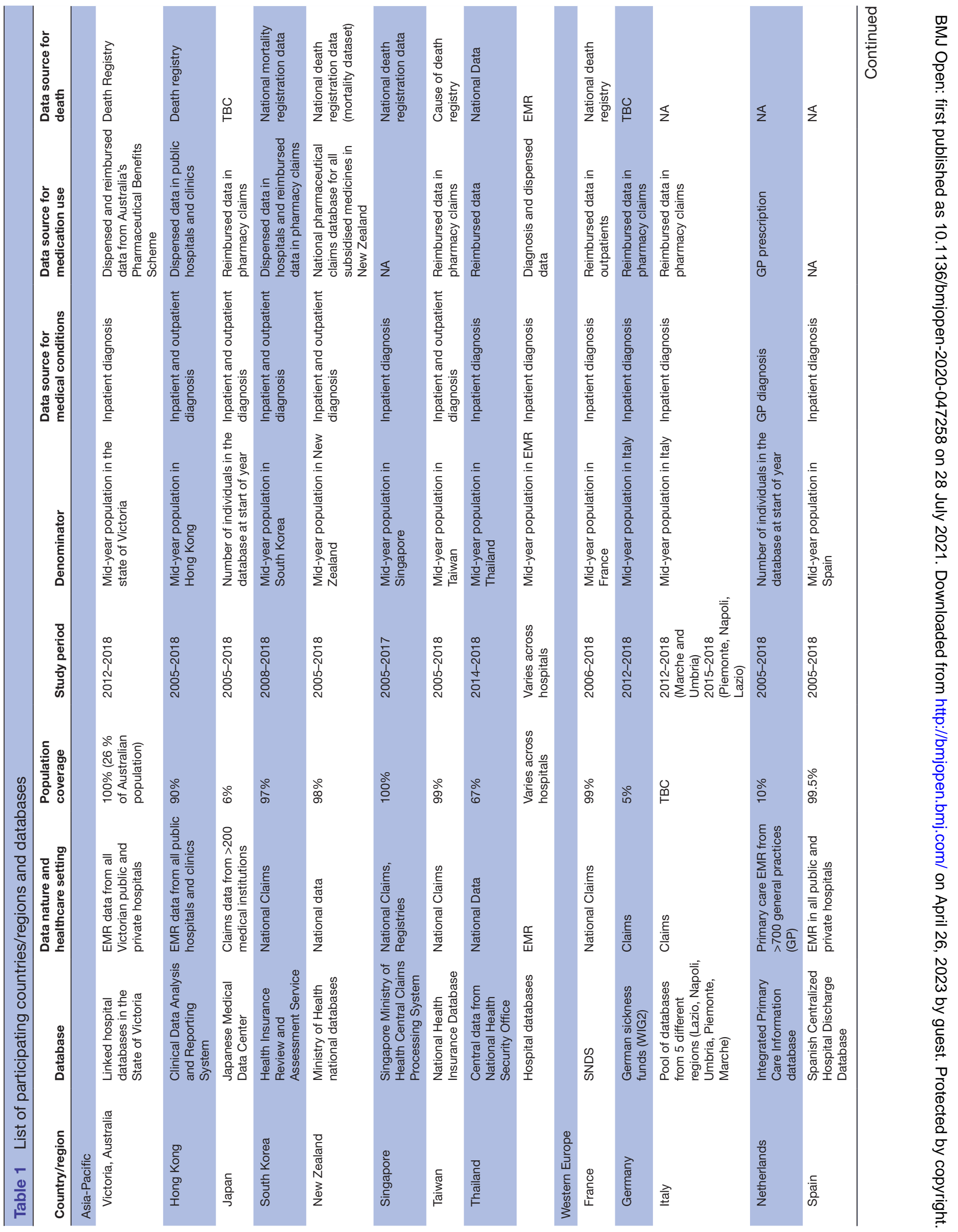




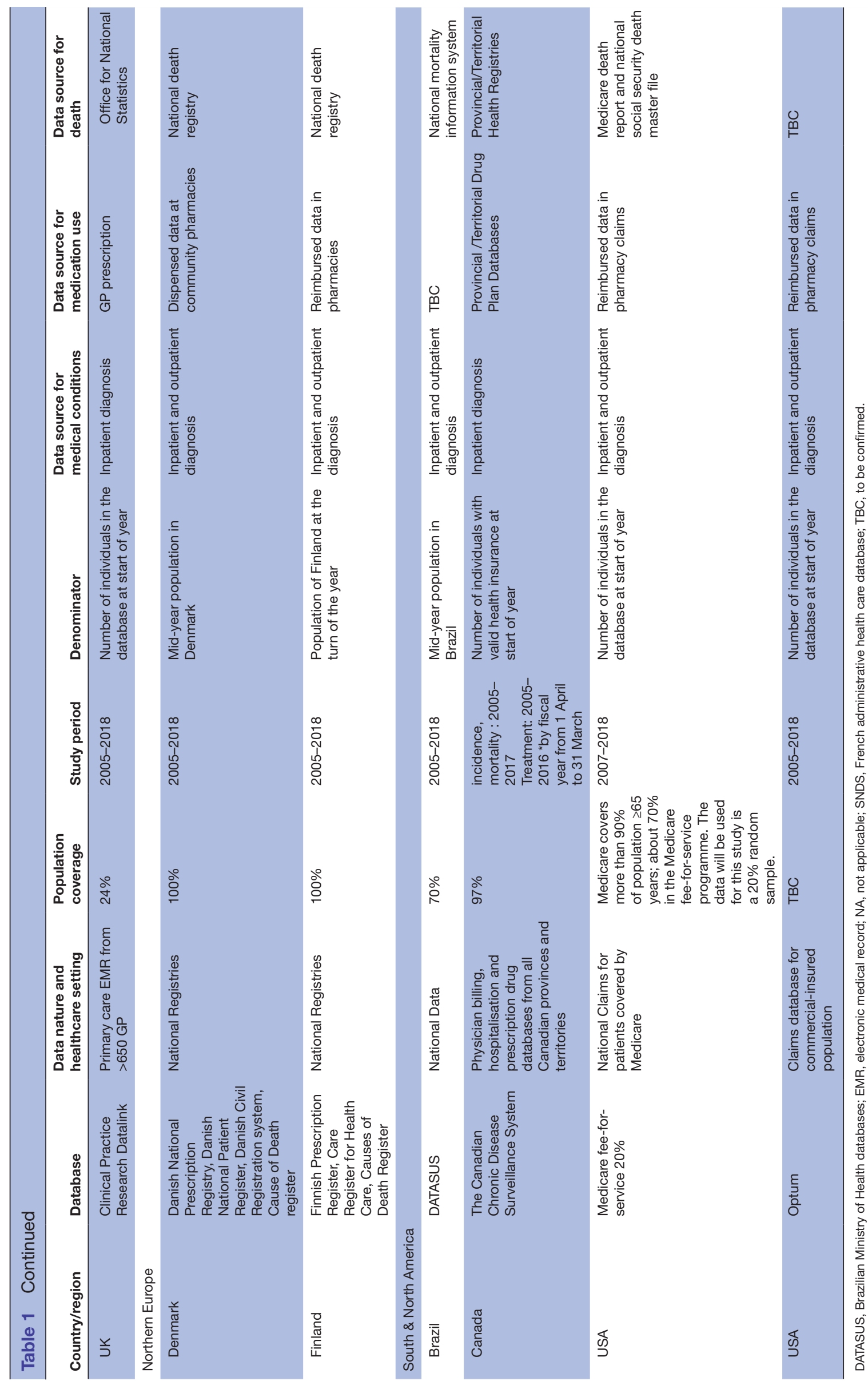

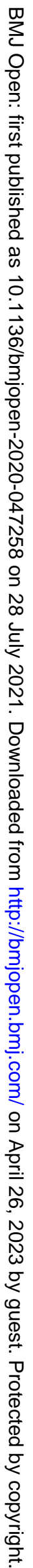


contribute the treatment rates for objective 2. Study sites can contribute the mortality rates for objective 3 if their database contains death data or can link to death registries.

\section{Study population}

Patients aged 50 years and older and hospitalised due to hip fracture from 1 January 2005 to 31 December 2018 will be included. We use 50 years old as a cut-off age because women generally enter menopause at 50 years old and their risk of osteoporosis and fractures after then increases. ${ }^{1116}$ Patients will be excluded if they meet any of the following criteria: (1) had a diagnosis of hip fracture within 12 months before the initial hip fracture; (2) had missing sex or age information; or (3) had less than 12 months continuous observation period in the data source prior to the start of the calendar year.

Identification of the 12 months observation period in the data source depends on the type of data source. For a database of medical claims, the patient's enrolment date should precede the hip fracture by at least 12 months. For a database of hospital electronic medical records, the patient's first event (eg, medical visit or prescription) in the database should precede the hip fracture by at least 12 months.

\section{Baseline and follow-up period}

The index date will be defined as the date of admission for the initial hip fracture. The baseline period will be the 1-year period before the index date (not including the index date).

For the primary objective of hip fracture incidence, there is no follow-up of patients. For the secondary objectives of post-fracture pharmacological treatment and mortality, each patient will be followed from the index date until another hip fracture episode, 12 months, death, disenrollment from database, 31 December 2019 or the end of data availability in a database, whichever is earliest.

\section{Outcome assessment}

Hip fracture episodes will be defined as an in-patient diagnosis with ICD-9/-10 codes or equivalent codes of other diagnostic coding systems. Hip fracture is a major clinical event that almost always requires hospitalisation and is generally accurately coded. ${ }^{17-19}$ The diagnosis codes to identify hip fracture are subject to local clinical practice; the sites will use their own standard or validated algorithms for identifying hip fracture. The algorithms for hip fracture used by each site, and positive predictive values where available, are provided in table 2. Most data sources have inpatient data. If inpatient diagnoses are not available, for example, in databases from general practice (eg, Netherlands), the documented hip fracture will be used. Patients may have multiple hip fracture episodes during the study period. The initial hip fracture will be defined as the first occurrence of hip fracture without any inpatient or outpatient hip fracture diagnosis during the 1-year baseline period. All the hip fracture episodes including the initial hip fracture and any subsequent new episodes (contralateral or ipsilateral) will be considered in the calculation of hip fracture incidence. Subsequent new episodes are defined by no inpatient hip fracture diagnosis in the 180 days prior (ie, wash-out period). A study design schema for defining hip fracture episodes is illustrated in figure 1.

Pharmacological treatments for fracture prevention include medications that are recommended for secondary prevention of osteoporotic fractures. These medications will be identified with prescription/dispensing of the medications classified using the WHO Anatomical Therapeutic Chemical Classification System codes whenever possible or equivalent codes of other drug coding systems used at the study site.

Date or month of death will be extracted. The cause of death (defined by ICD-9/-10 codes, or equivalent codes of other classification systems used at the study sites) will be included if available.

\section{Covariate assessment}

Sex and date or month of birth (or age at index date) will be captured. In addition, history of osteoporosis treatment defined as at least one prescription/dispensing record of any antiosteoporosis medication during the 1-year baseline period will be captured.

For the secondary objective of treatment following hip fracture, patients will be considered as 'ever use' if the patient had a history of osteoporosis treatment; and patient will be considered as 'new use' if the patient did not have a history of osteoporosis treatment.

\section{Statistical analysis}

Microsoft Excel, R, Statistical Analysis System (SAS) will be used for data management and analyses. The proportion of missing data will be reported, but missing data will not be imputed. Patients with missing age or sex information will be excluded during the selection procedure. The number of study variables collected per patient is small and the impact of missing data is expected to be minimal and not likely to impact the reliability of the results.

\section{Description of patient characteristics}

Description of baseline characteristics will include age, sex and history of antiosteoporosis medications. Discrete variables will be summarised using frequencies and proportions, and continuous variables will be summarised using means and SD or medians and IQR, as appropriate. Age will be categorised into 5-year age bands: 50-54, 55-59, $60-64,65-69,70-74,75-79,80-84,85$ or above.

\section{Primary objective: incidence of hip fracture}

Population data will be used as the denominator (ie, population at risk) to calculate the annual incidence of hip fracture. The population of each calendar year will be defined as people (1) aged 50 years and older, (2) with known sex, (3) enrolled/registered in the database on 1 January of that year, and (4) with a 1-year baseline 


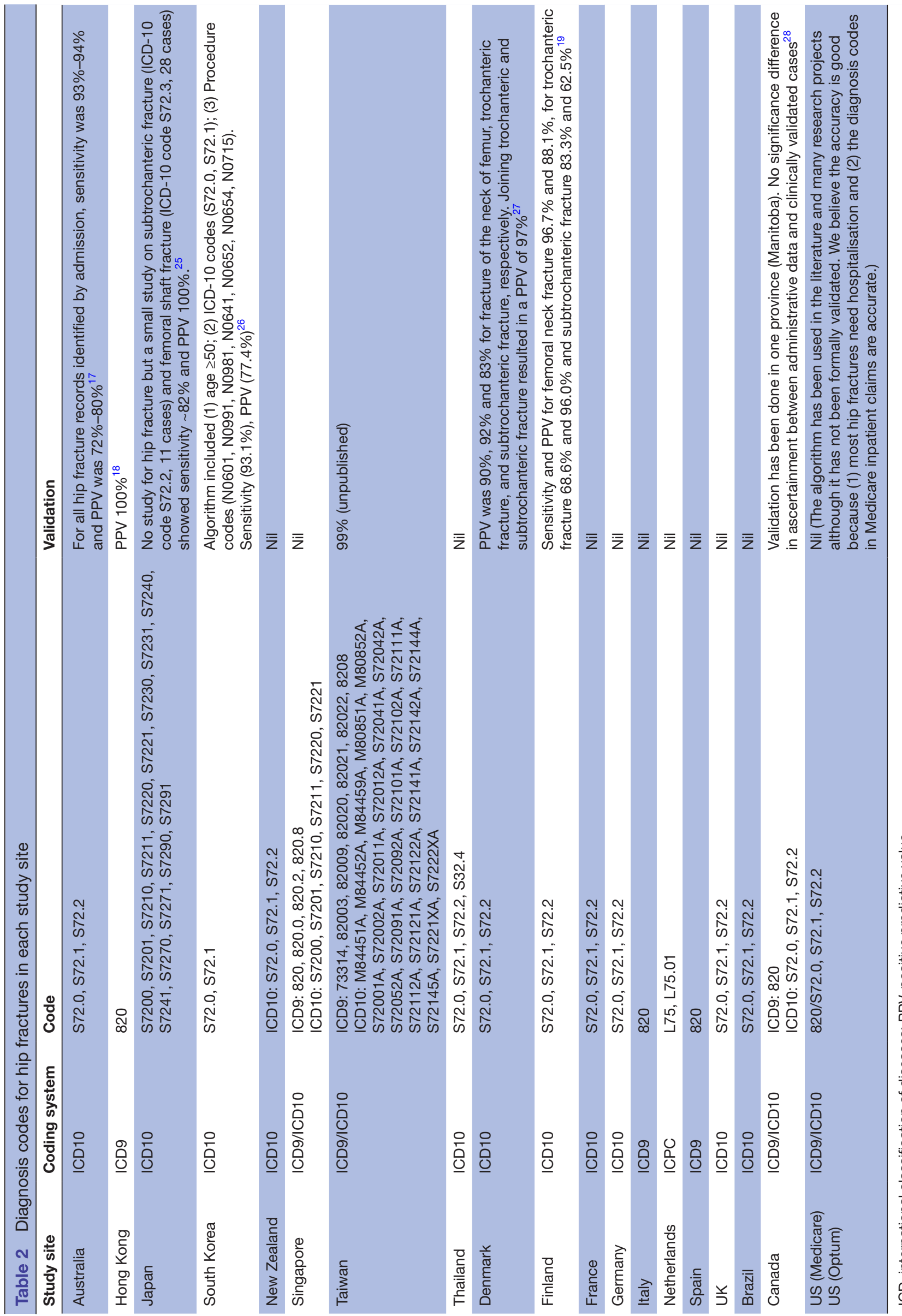


Study period: 01 Jan 2005 to 31 Dec 2018

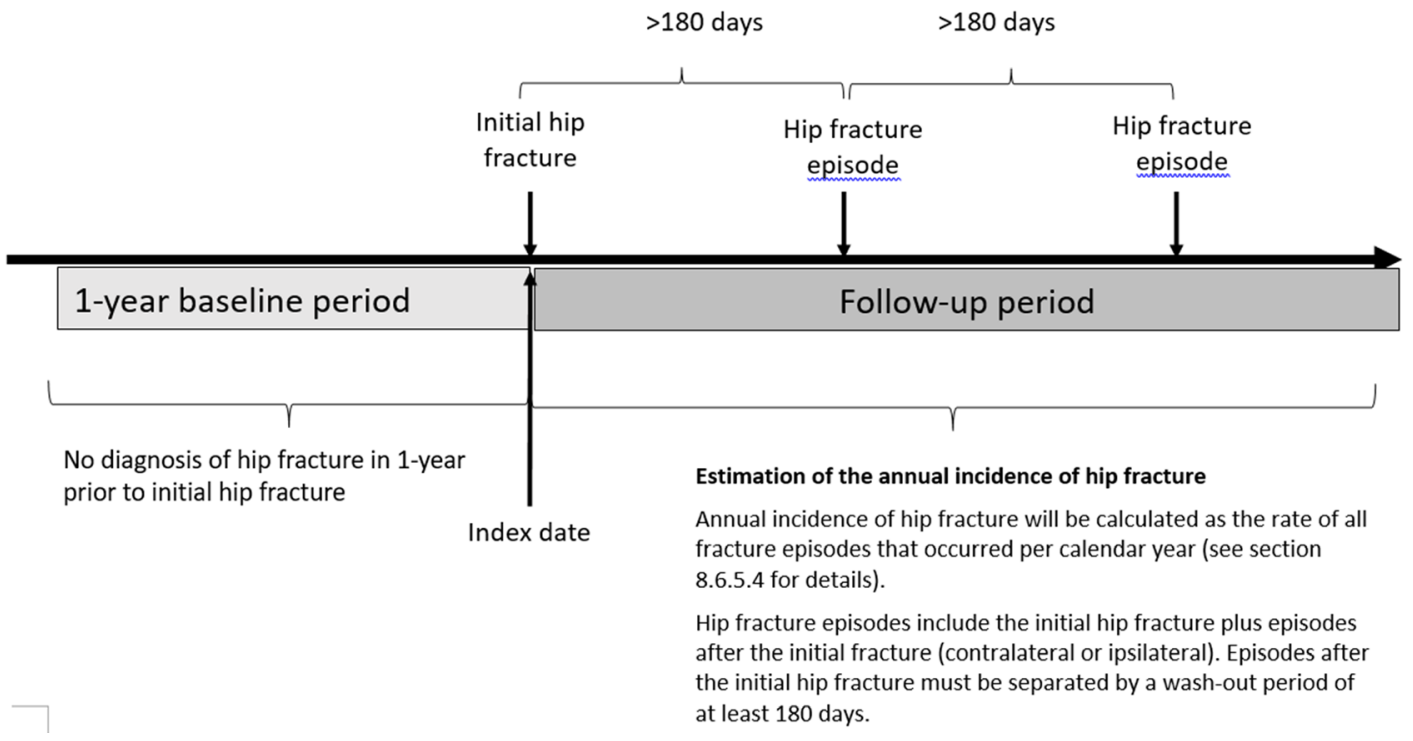

Figure 1 Study design schema for estimating incidence of hip fracture (objective 1).

period. If the population in the database is unknown, the national/regional population reported by the government will be used. The mid-year population of the database or the reported national/regional population aged 50 years and older of each calendar year will be used as the denominator.

Similar to prior studies, ${ }^{20-22}$ the incidence (per 100000 persons) rate per calendar year of hip fractures will be calculated as the sum of new hip fracture episodes in a year divided by the population at risk on 1 January of that year. In addition, age-standardised and sex-standardised incidence will be calculated to facilitate cross-geographical comparisons. The world population estimates in 2020 reported by the United Nations (https://population. un.org/wpp/Download/Standard/Population/) will be used as a standard.

A linear regression model will be used to test for time trends in the annual incidence in each site, assuming a linear trend for the hip fracture incidence, throughout the study period. The annual incidence as a dependent variable and the calendar year as a predictor variable will be fitted into the model. A two-tailed $\mathrm{p}<0.05$ will be considered statistically significant.

\section{Secondary objective: treatment proportion}

Similar to a prior study, ${ }^{23}$ we will use the Kaplan-Meier method to estimate the treatment proportion within 3,6 and 12 months of fracture and 95\% CI, censoring patients on another hip fracture episode, 12 months, death, disenrolment from database, 31 December 2019, or the end of data availability in a database; whichever is earliest.

The description of the treatment proportion will include (1) the treatment proportion by year of initial hip fracture; (2) the treatment proportion for new medication users (treatment-naive), defined as those with no prescription filled for osteoporosis medications within 12 months prior to their hip fracture (ie, during the baseline period); and (3) the treatment proportion by the type of treatment (see table 3 for details).

Secondary objective: one-year mortality following hip fracture Similar to a prior study, ${ }^{21}$ the 1 -year mortality (per 100 patients) rate per calendar year of initial hip fracture will be calculated as the sum of patients who died of any cause during the 12-month follow-up period divided by the sum of patients with an initial hip fracture. An additional

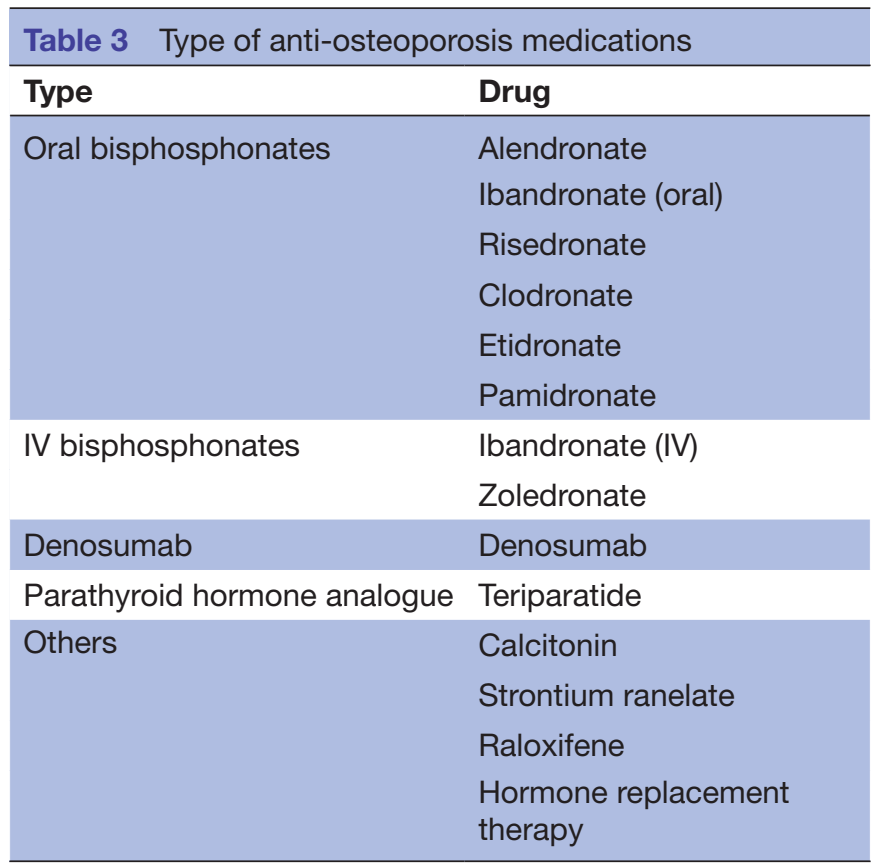


analysis using the Kaplan-Meier method to account for censoring will be included as well. In addition, the mortality will be ascertained for the first 3 months and the first 6 months after the initial hip fracture. Agestandardised and sex-standardised mortality will be calculated to facilitate cross-geographical comparisons. The world population estimates in 2020 reported by the United Nations will be used as a standard.

\section{Additional analysis}

Sensitivity analyses will be performed to evaluate the robustness of the results from the primary analysis. In the primary analysis, a wash-out period of 180 days is used to define a new episode of hip fracture. In the sensitivity analysis, a shorter (90 days) and a longer (365 days) wash-out period will be used. In addition, the requirement of at least 12-month continuous observation period may not capture fractures in a given year among those with less than a year of prior observation. Thus, a sensitivity analysis by removing this requirement will be conducted to evaluate if this requirement affects the estimates.

Fractures may occur in patients for reasons other than osteoporosis. In databases where the information is available, we will repeat the analysis in the subgroup excluding patients with any of the following criteria: (1) concurrent diagnosis of high trauma fractures (high trauma is defined as vehicle accident or fall from greater than standing height); (2) bone metastasis during the 1-year baseline period; (3) Paget's disease during the 1-year baseline period; or (4) osteogenesis imperfecta during the 1-year baseline period.

Given the high mortality in the first year after hip fracture, death could be a competing risk event leading to overestimation of treatment probability. Therefore, a competing risk analysis using the cumulative incidence function approach will be performed to estimate the marginal probability of treatment with adjustment for competing risk of death.

Age-specific and sex-specific estimates of hip fracture incidence and mortality will be provided in 5 years age bands: 50-54, 55-59, 60-64, 65-69, 70-74, 75-79, 80-84, 85 or above.

\section{Analytical common data model}

The ACDM will be created to increase validity and consistency of data analysis using multidatabases. The sites will convert de-identified subject-level data into table formats in ACDM and use standard programming codes to conduct the statistical analysis and generate aggregatelevel data. The ACDM will be codeveloped by HKU and Amgen. $R$ and SAS programming codes will be developed by the programming team in HKU and Amgen, respectively. To ensure quality assurance, at least two programmers will be involved to cross check the codes. The R and SAS programming codes will run on the same sample dataset and the results should be a $100 \%$ match. It is expected that the development of ACDM and programming codes will take around 2-3 months. Since the data structure varies across databases, HKU will discuss with the sites if any modification of the ACDM and programming codes will be needed. All the site-specific modifications will be documented. Sharing of the script as open-source code will be subject to journal requirement when the results are published.

\section{Sample size}

The estimated sample size in the databases ranges from several hundred hip fractures per year to tens of thousands of hip fractures per year. For example, the data source for Hong Kong, a region of 7.2 million people with 2.8 million adults aged 50+, has approximately 9300 hip fractures per year in adults aged $50+$ (a crude rate of 330 fractures per 100000 ). The estimated samples sizes for each database are provided in table 4.

\section{Limitations}

In general, most of the databases were built for administrative or reimbursement purposes rather than research purposes. The databases represent a variety of data sources, healthcare settings, and coding practices each of which will have different features and limitations. The strengths and limitations of different type of databases have been discussed elsewhere. ${ }^{24}$ The features of the databases in this study are shown in table 1. A majority of databases have a high (over 90\%) population coverage and official census data will be used as denominator. Databases with lower population coverage will use the actual number of individuals in the databases as denominator (Japan, UK and USA). The databases in Italy do not link to national/regional death registry. National prescription data are only available in Australia, Denmark, Finland, New Zealand, South Korea and Taiwan.

\section{Measurement errors/misclassifications}

The study will use prescription/dispensing data to assess treatment, which is only a proxy for the patient taking their medication. The actual treatment with certain medications, such as oral bisphosphonates, may therefore be overestimated. In addition, use of zoledronic acid is not expected to be captured in all databases. For example, in countries where zoledronic acid is administered in hospitals or outpatient clinics, some databases do not readily capture medication administered in the hospital setting. In such circumstances, patients may be misclassified as having no treatment even though they were exposed to zoledronic acid.

The database for Hong Kong does not capture clinical records from private clinics/hospitals, though it is expected that most of the cases will be admitted to public hospitals via emergency service.

Several databases will capture only treatments in the public reimbursement system (Hong Kong, South Korea, Taiwan and others); hence, the treatment rates might be underestimated by not including patients in the private payment system. Similarly, non-reimbursed medications 


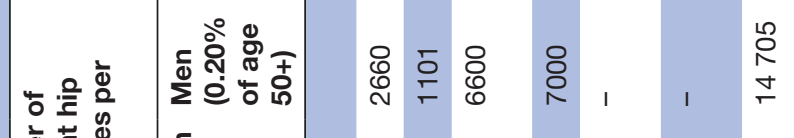

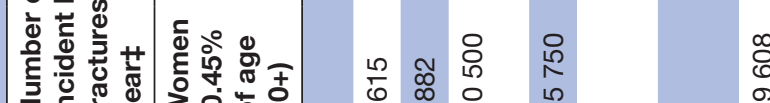

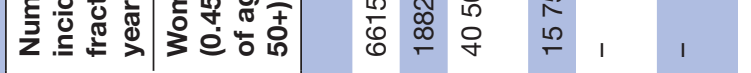

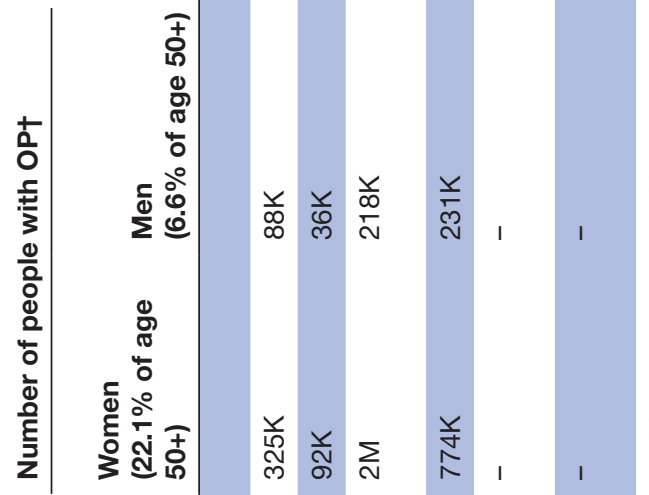

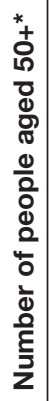

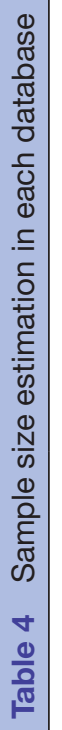

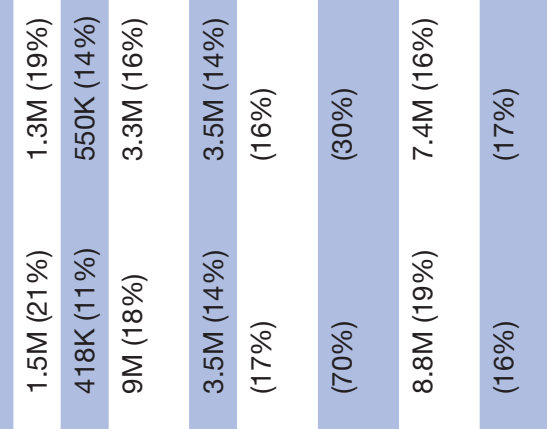

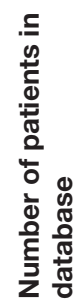

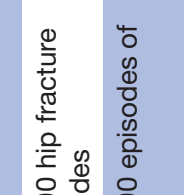

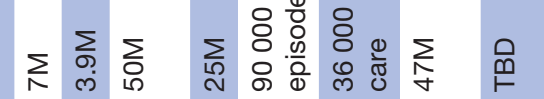

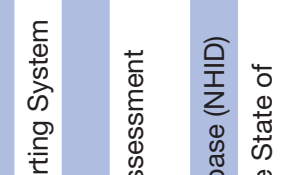

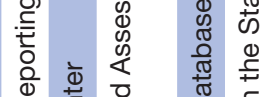

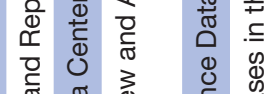

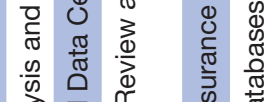

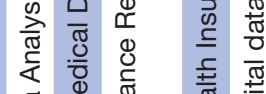

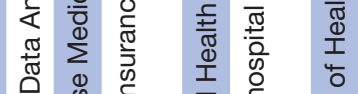

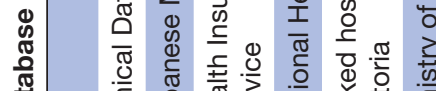

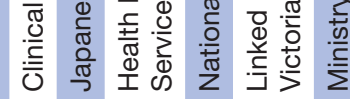

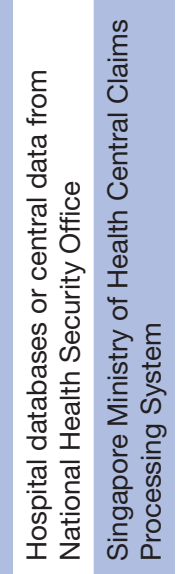

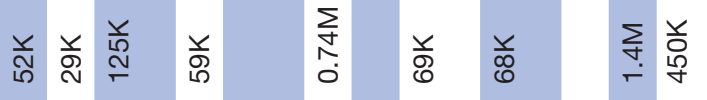

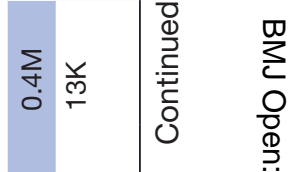

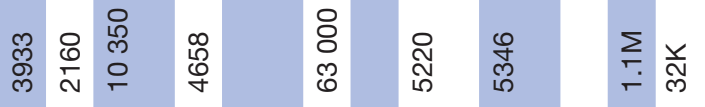

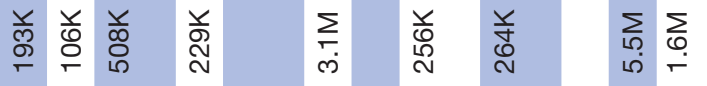

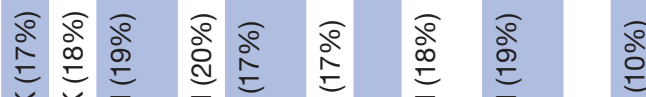

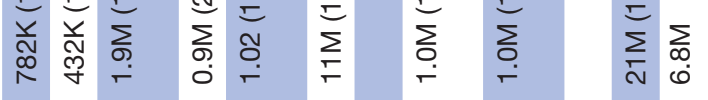

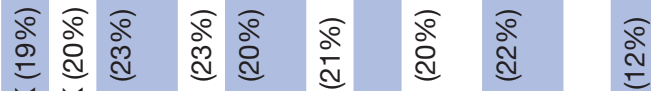

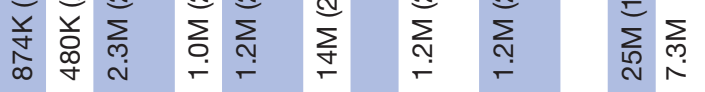

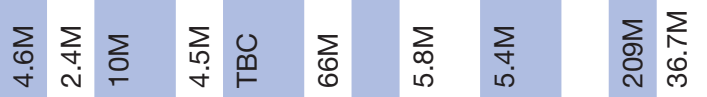

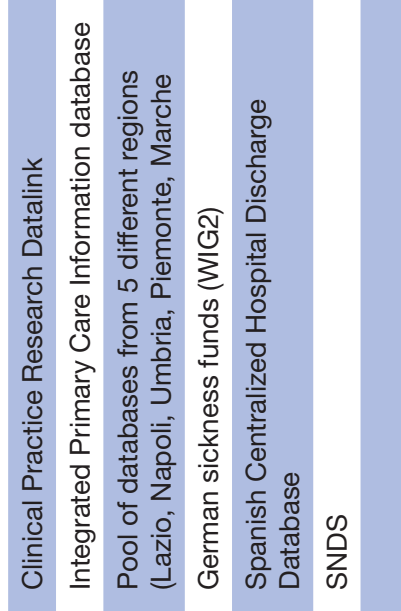

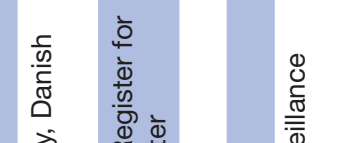

$\overrightarrow{\vec{B}}$

음

$\frac{\sqrt{0}}{\stackrel{0}{\circ}}$

ڤ

$\overrightarrow{\vec{\omega}}$

을.

Ñ

家

N్

옥

N

$\stackrel{\varrho}{\varrho}$

N

익

혹.

옳

웅

N

N

इ

잉

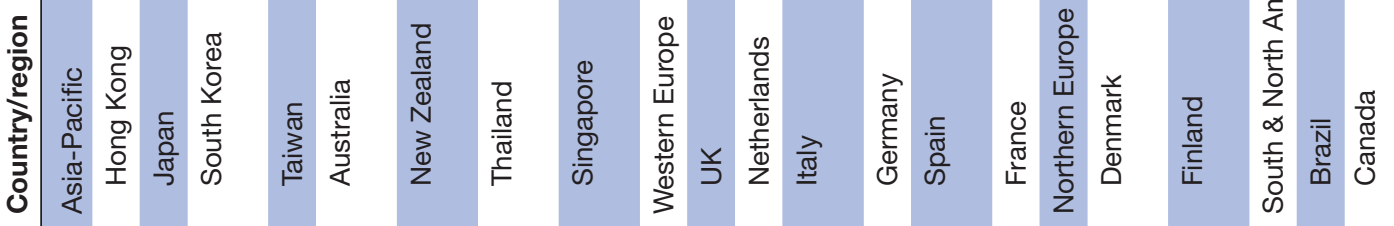




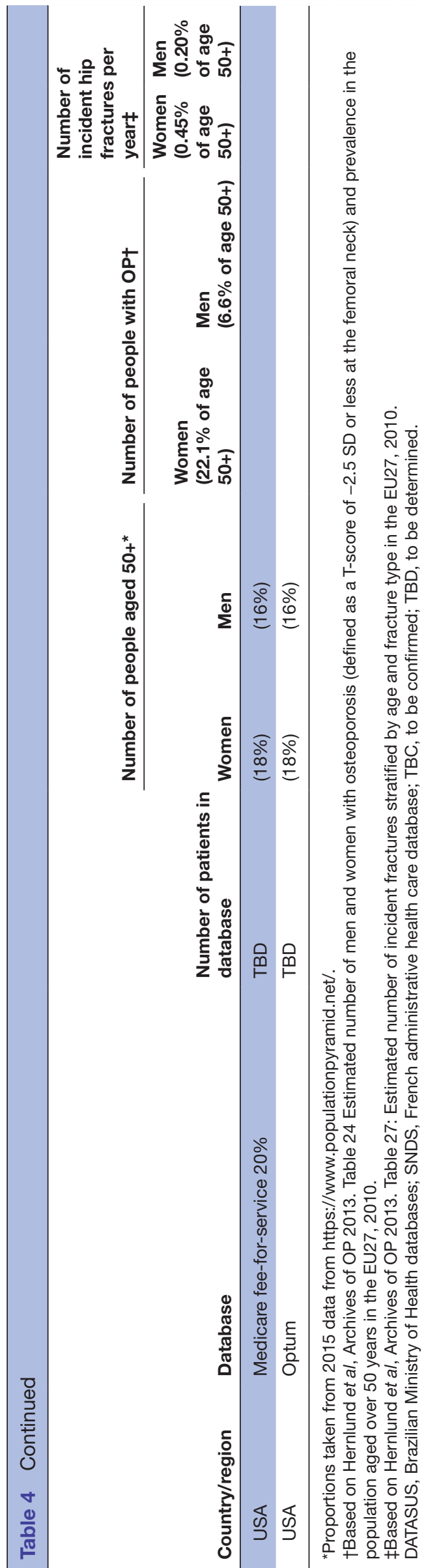

cannot be captured in the reimbursement system, leading to potential underestimation of treatment rates.

Information bias

Since hip fracture is a major clinical event that almost always requires hospitalisation, only hospital diagnoses of hip fracture will be considered in most databases (except when inpatient diagnoses are not available in the database). Fractures may occur in patients for reasons other than osteoporosis (eg, trauma, bone metastasis, Paget's disease, osteogenesis imperfect). Eligibility criteria for the study have been kept broad for the practical purpose of applying consistent definitions across multiple databases. To inform interpretation, we will conduct a sensitivity analysis excluding patients with these four criteria in those databases able to support the analysis.

\section{Selection bias}

All patients who fulfil the eligibility criteria in each database will be included. A majority of the databases cover over $90 \%$ of the population (eg, Finland, Hong Kong, South Korea and others; see table 1) and therefore selection bias is not expected to be a major issue in these databases. However, a few data sources will be representative of local hospitals with limited population coverage (eg, Thailand), leading to potential selection bias. For instance, the Japanese database has no subjects aged $75+$ years and limited number of subjects aged $>60$ years compared with national statistics. Given that the Japan data source does not contain the oldest adults at highest risk for hip fracture, the current protocol will provide an underestimation of the overall population incidence of hip fracture in Japan. However, the age subgroup analysis will provide a reasonable measure of incidence in the population under 75 years old. Therefore, we will exclude Japan data in the estimation of overall population incidence but will include it only in the age-specific analysis. Although these sites have limited data for population estimates, the results are still informative for crossgeographical comparisons. More importantly, the site participation in this study can facilitate global cooperation, and also raise the awareness of the need for standardised high-quality national data for research.

\section{Patient and public involvement}

The study will involve retrospective analysis of secondary data collected from databases. Patients are deidentified and there is no direct patient involvement. However, several researchers involved in this study routinely consult with patients in the design, development and reporting of research at a national level. Patients may be involved in presentations and dissemination of the results at a national level. Each participating site will be responsible for obtaining ethical clearances in accordance with current regulations within their local jurisdiction.

\section{Ethics and dissemination}

Each participating site will follow the relevant local ethics and regulatory frameworks for study approval. The status 
of ethics approval in each site is listed in online supplemental table. All data to be used in this study are taken from existing anonymised records. In addition, participating sites will only share aggregated data with the study coordinators.

The results of the study will be submitted for peerreviewed scientific publications and presented in scientific conferences. Authorship of any publications resulting from this study will be determined on the basis of the International Committee of Medical Journal Editors Recommendations for the Conduct, Reporting, Editing, and Publication of Scholarly Work in Medical Journals.

\section{Author affiliations}

${ }^{1}$ Department of Pharmacology and Pharmacy, Li Ka Shing Faculty of Medicine, University of Hong Kong, Hong Kong, Hong Kong SAR

${ }^{2}$ Center for Observational Research, Amgen Inc, Thousand Oaks, California, USA

${ }^{3}$ Centre for Surveillance and Applied Research, Public Health Agency of Canada, Ottawa, Ontario, Canada

${ }^{4}$ Centre for Medicine Use and Safety, Faculty of Pharmacy and Pharmaceutical Sciences, Monash University, Melbourne, Victoria, Australia

${ }^{5}$ School of Pharmacy, The University of Auckland, Auckland, New Zealand

${ }^{6}$ Bordeaux PharmacoEpi, University of Bordeaux, Bordeaux, France

${ }^{7}$ Osteoporosis and Bone Metabolism Unit, Department of Endocrinology, Singapore

General Hospital, Singapore

${ }^{8}$ Ministry of Health Singapore, Singapore

${ }^{9}$ School of Pharmacy, University of Eastern Finland, Kuopio, Finland

${ }^{10}$ School of Pharmacy, Sungkyunkwan University, Suwon, South Korea

${ }^{11}$ Hinda and Arthur Marcus Institute for Aging Research, Hebrew SeniorLife and Department of Medicine, Beth Israel Deaconess Medical Center, Harvard Medical School, Boston, Massachusetts, USA

${ }^{12} \mathrm{NPO}$ Drug Safety Research Unit Japan, Tokyo, Japan

${ }^{13}$ School of Pharmacy, Institute of Clinical Pharmacy and Pharmaceutical Sciences, National Cheng Kung University, Tainan, Taiwan

${ }^{14}$ University of New Mexico School of Medicine, Albuquerque, New Mexico, USA

${ }^{15}$ Chronic Disease Research Group, Hennepin Healthcare Research Institute,

Minneapolis, Minnesota, USA

${ }^{16}$ Research Department of Practice and Policy, University College London School of

Pharmacy, London, UK

${ }^{17}$ Centre for Medicines Optimisation Research and Education (CMORE), University

College London Hospital, London, UK

${ }^{18}$ Rheumatology Service, Internal Medicine Department, School of Medicine, Universidade Federal do Rio de Janeiro, Rio de Janeiro, Brazil

${ }^{19}$ School of Pharmacy, The Nihon University, Chiba, Japan

${ }^{20}$ Department of Clinical Epidemiology, Aarhus University Hospital, Aarhus University, Aarhus, Denmark

${ }^{21}$ Nuffield Department of Orthopaedics, Rheumatology, and Musculoskeletal

Sciences, University of Oxford, Oxford, UK

${ }^{22}$ School of Public Health, National University of Singapore, Singapore

${ }^{23}$ Department of Medical Informatics, Erasmus Medical Center, Rotterdam, Netherlands

${ }^{24}$ Faculty of Pharmaceutical Sciences, Ubon Ratchathani University, Mueang Si Khai, Ubon Ratchathani, Thailand

${ }^{25}$ Shanghai Synyi Medical Technology Co Ltd, Shanghai, China

Twitter Kenneth K C Man @KennethKCMan and Mirhelen Mendes de Abreu @ Mirhelen

Contributors C-WS codeveloped and wrote the protocol. T-CL codeveloped, drafted, reviewed and commented on the protocol. JO, CB, JeL, C-LC and KKCM codeveloped, reviewed and commented on the protocol. SB, JSB, KB, PB-L, AHYC, MC, CYD, CD-P, GG, SH, JI, HEJ, DPK, KK, EC-CL, EML, JiL, MMdA, NM, NO, ABP, DPA, J-YS, HTS, KBT, A-MT, KMCV, GH-MW, SW and HZ reviewed and commented on the protocol. ICKW is the principal investigator of the study, takes responsibility for the integrity of the study, codeveloped, reviewed and commented on the protocol.

Funding This work was supported by Amgen Inc. Award/Grant number is not applicable.
Competing interests ICKW, SW, KMCV, A-MT, HTS, J-YS, DP-A, MMdA, EC-CL, KK, CD-P, MC, AHYC, and JSB had financial support from Amgen Inc. for the submitted work.

Patient consent for publication Not required.

Provenance and peer review Not commissioned; externally peer reviewed.

Supplemental material This content has been supplied by the author(s). It has not been vetted by BMJ Publishing Group Limited (BMJ) and may not have been peer-reviewed. Any opinions or recommendations discussed are solely those of the author(s) and are not endorsed by BMJ. BMJ disclaims all liability and responsibility arising from any reliance placed on the content. Where the content includes any translated material, BMJ does not warrant the accuracy and reliability of the translations (including but not limited to local regulations, clinical guidelines, terminology, drug names and drug dosages), and is not responsible for any error and/or omissions arising from translation and adaptation or otherwise.

Open access This is an open access article distributed in accordance with the Creative Commons Attribution Non Commercial (CC BY-NC 4.0) license, which permits others to distribute, remix, adapt, build upon this work non-commercially, and license their derivative works on different terms, provided the original work is properly cited, appropriate credit is given, any changes made indicated, and the use is non-commercial. See: http://creativecommons.org/licenses/by-nc/4.0/.

\section{ORCID iDs}

Chor-Wing Sing http://orcid.org/0000-0002-2180-3676

Kenneth K C Man http://orcid.org/0000-0001-8645-1942

Mirhelen Mendes de Abreu http://orcid.org/0000-0002-2264-0442

Daniel Prieto-Alhambra http://orcid.org/0000-0002-3950-6346

Ju-Young Shin http://orcid.org/0000-0002-2791-1037

Katia M C Verhamme http://orcid.org/0000-0001-8162-4904

Hongxin Zhao http://orcid.org/0000-0001-7906-2797

\section{REFERENCES}

1 Ballane G, Cauley JA, Luckey MM, et al. Secular trends in hip fractures worldwide: opposing trends East versus West. J Bone Miner Res 2014;29:1745-55.

2 Johnell O, Kanis JA. An estimate of the worldwide prevalence, mortality and disability associated with hip fracture. Osteoporos Int 2004;15:897-902.

3 Downey C, Kelly M, Quinlan JF. Changing trends in the mortality rate at 1-year post hip fracture - a systematic review. World J Orthop 2019;10:166-75.

4 Kanis JA, Odén A, McCloskey EV, et al. A systematic review of hip fracture incidence and probability of fracture worldwide. Osteoporos Int 2012;23:2239-56.

5 Balasubramanian A, Zhang J, Chen L, et al. Risk of subsequent fracture after prior fracture among older women. Osteoporos Int 2019;30:79-92.

6 ASBMR. Secondary fracture prevention initiative. coalition clinical recommendation, 2018. Available: https://www.secondaryfractures. org/clinical-recommendations/ [Accessed Sep 2019].

7 Kanis JA, Cooper C, Rizzoli R, et al. European guidance for the diagnosis and management of osteoporosis in postmenopausal women. Osteoporos Int 2019;30:3-44.

8 Solomon DH, Johnston SS, Boytsov NN, et al. Osteoporosis medication use after hip fracture in U.S. patients between 2002 and 2011. J Bone Miner Res 2014;29:1929-37.

9 Kim SC, Kim M-S, Sanfélix-Gimeno G, et al. Use of osteoporosis medications after hospitalization for hip fracture: a cross-national study. Am J Med 2015;128:519-26.

10 Desai RJ, Mahesri M, Abdia Y, et al. Association of osteoporosis medication use after hip fracture with prevention of subsequent Nonvertebral fractures: an instrumental variable analysis. JAMA Netw Open 2018;1:e180826.

11 Hernlund E, Svedbom A, Ivergård M, et al. Osteoporosis in the European Union: medical management, epidemiology and economic burden. A report prepared in collaboration with the International osteoporosis Foundation (IOF) and the European Federation of pharmaceutical industry associations (EFPIA). Arch Osteoporos 2013;8:136.

12 Reginster JY, Neuprez A, Dardenne N, et al. Efficacy and safety of currently marketed anti-osteoporosis medications. Best Pract Res Clin Endocrinol Metab 2014;28:809-34.

13 Khosla S, Cauley JA, Compston J, et al. Addressing the crisis in the treatment of osteoporosis: a path forward. J Bone Miner Res 2017;32:424-30. 
14 Cooper C, Cole ZA, Holroyd CR, et al. Secular trends in the incidence of hip and other osteoporotic fractures. Osteoporos Int 2011;22:1277-88.

15 Lai EC-C, Ryan P, Zhang Y, et al. Applying a common data model to Asian databases for multinational pharmacoepidemiologic studies: opportunities and challenges. Clin Epidemiol 2018;10:875-85.

16 Camacho PM, Petak SM, Binkley N, et al. American association of clinical endocrinologists/american College of endocrinology clinical practice guidelines for the diagnosis and treatment of postmenopausal osteoporosis-2020 update. Endocr Pract 2020;26:1-46.

17 Thuy Trinh LT, Achat H, Loh SM, et al. Validity of routinely collected data in identifying hip fractures at a major tertiary hospital in Australia. Health Inf Manag 2018;47:38-45.

18 Sing C-W, Woo Y-C, Lee ACH, et al. Validity of major osteoporotic fracture diagnosis codes in the clinical data analysis and reporting system in Hong Kong. Pharmacoepidemiol Drug Saf 2017;26:973-6.

19 Sund R, Nurmi-Lüthje I, Lüthje P, et al. Comparing properties of audit data and routinely collected register data in case of performance assessment of hip fracture treatment in Finland. Methods Inf Med 2007;46:558-66.

20 Lewiecki EM, Wright NC, Curtis JR, et al. Hip fracture trends in the United States, 2002 to 2015. Osteoporos Int 2018;29:717-22.
21 Brauer CA, Coca-Perraillon M, Cutler DM, et al. Incidence and mortality of hip fractures in the United States. JAMA 2009;302:1573-9.

22 Chau $\mathrm{PH}$, Wong $\mathrm{M}$, Lee $\mathrm{A}$, et al. Trends in hip fracture incidence and mortality in Chinese population from Hong Kong 2001-09. Age Ageing 2013;42:229-33.

23 Cadarette SM, Katz JN, Brookhart MA. Trends in drug prescribing for osteoporosis after hip fracture, 1995-2004. J Rheumatol 2008;35:319-26.

24 Schneeweiss S, Avorn J. A review of uses of health care utilization databases for epidemiologic research on therapeutics. J Clin Epidemiol 2005;58:323-37.

25 Tanaka S, Hagino H, Ishizuka A. Validation study of claims-based definitions of suspected atypical femoral fractures using clinical information. Jpn J Pharmacol/Yakuzai ekigaku 2016;21:13-19.

26 Park C, Jang S, Jang S, et al. Identification and validation of osteoporotic hip fracture using the National health insurance database. J Korean Hip Soc 2010;22:305-11.

27 Hjelholt TJ, Edwards NM, Vesterager JD, et al. The positive predictive value of hip fracture diagnoses and surgical procedure codes in the Danish multidisciplinary hip fracture registry and the Danish national patient registry. Clin Epidemiol 2020;12:123-31.

28 Lix LM, Azimaee M, Osman BA, et al. Osteoporosis-related fracture case definitions for population-based administrative data. BMC Public Health 2012;12:301. 